\title{
Mesenteric Paraganglioma-A Cyst in The Mist
}

\author{
Saniha. P.B, M.N. Jadhav* and S.K. Kittur \\ Department of Pathology, Belagavi Institute of Medical Sciences, Belagavi-590001 INDIA
}

\begin{abstract}
Extraadrenal paraganglioma is rarely found in the mesentery. It accounts only for $5-10 \%$ of cases and may present incidentally as abdominal mass. We report a case of 30 year old male presented as mass per abdomen. A differential diagnosis of neurogenic tumor or cystic mesothelioma was considered based on CT-abdomen. Histopathological examination showed features of mesenteric paraganglioma and confirmed on immunohistochemistry. This case report highlighted several important key issues regarding diagnosis of paraganglioma, including malignant or metastatic potential, recurrence rate, pre and postoperative imaging study and lifelong follow-up of the patient.
\end{abstract}

\section{Keywords: Cyst, Extraadrenal, Mesentery, Paraganglioma.}

\section{Introduction}

Pheochromocytoma arising outside the adrenal gland from neuroendocrine cells is called paraganglioma. When it occurs below the diaphragm, in the organ of Zuckerkandl or retroperitoneum, it is called as extraadrenal paraganglioma. ${ }^{[1]}$ It accounts only for $5-10 \%$ of cases and extend anywhere from the neck down to the base of pelvis. ${ }^{[2]}$ Extraadrenal paragangliomas are rarely found in the mesentery. They typically present as solid tumors and infrequently detected as cystic tumors. The literature search showed only 12 cases of mesenteric paragangliomas reported till date.$^{[3]}$ Here we report a case of nonfunctional mesenteric paraganglioma presented as a cystic lesion.

\section{Case History}

A 30 year old male presented with mass per abdomen associated with pain of five months duration. It was insidious in onset and progressive in nature. The pain was localized to right lumbar and paraumbilical region, which increased after intake of food and decreased on taking rest. There was no history of headache or palpitations. The vital signs were within normal limits. Per abdomen examination showed tender mass present in right lumbar and paraumbilical region of size $10 \times 6$ $\mathrm{cm}$. All routine investigations were within normal limits. Serological tests for HBsAg, HIV I \& II, HCV antibodies were negative. CT-abdomen (contrast enhanced) showed $8.8 \times 6.5 \mathrm{~cm}$, multiseptated ovoid mesenteric cystic lesion with solid component, causing extrinsic compression of IVC and suggestive of neurogenic tumor or cystic mesothelioma(Fig 1a). There was no evidence of invasion of surrounding viscera.

On exploratory laparotomy, the cyst was located at the junction of stomach and duodenum. There was no ascites or mesenteric lymphadenopathy. The cyst was excised. Vital signs were stable during operation and postoperatively. Gross examination of the excised cystic lesion was encapsulated, dark-brown measuring 9x8x6 $\mathrm{cm}$ with smooth outer surface. On cut section $30 \mathrm{ml}$ of blood tinged material was drained from the multiloculated cyst with partly cystic and partly solid brown areas (Fig 1b,c).

Microscopy from solid areas showed tumor composed of cells arranged in the form of nests (Zellballen) separated by vascular stroma. These cells showed vesicular nuclei, prominent nucleoli with abundant basophilic granular cytoplasm. There was no evidence of melanin pigment. There were no abnormal mitotic figures or capsular and lymphovascular invasion (Fig 1d, e). These features were suggestive of mesenteric paraganglioma. On immunohistochemistry(IHC) tumor cells were positive for vimentin, synaptophysin, chromogranin with focal S-100 protein positivity in sustentacular cells (Fig 2a-d) and negative for pancytokeratin, Epithelial Membrane Antigen ( EMA),Transcription Factor Binding to IGHM Enhancer 3(TFE3), Muscle Specific Actin (MSA),Paired box gene(PAX8) and CD10(Fig 3a-f). Finally, a diagnosis of mesenteric paraganglioma was made. The postoperative 24 hour urine catecholamine estimation was adviced and was within normal limits. There was no evidence of metastasis or recurrence on follow-up of patient till date.

\section{Discussion}

Extraadrenal abdominal paragangliomas are rare. Age of onset is between 30 and 45 years with male preponderance. ${ }^{[4]}$ The pathogenesis of paraganglioma is not fully understood. Paragangliomas can be further divided into functioning and nonfunctioning based on their ability to secrete hormones. They may be either sporadic/ hereditary. Overall, as many as $10-50 \%$ of paragangliomas 


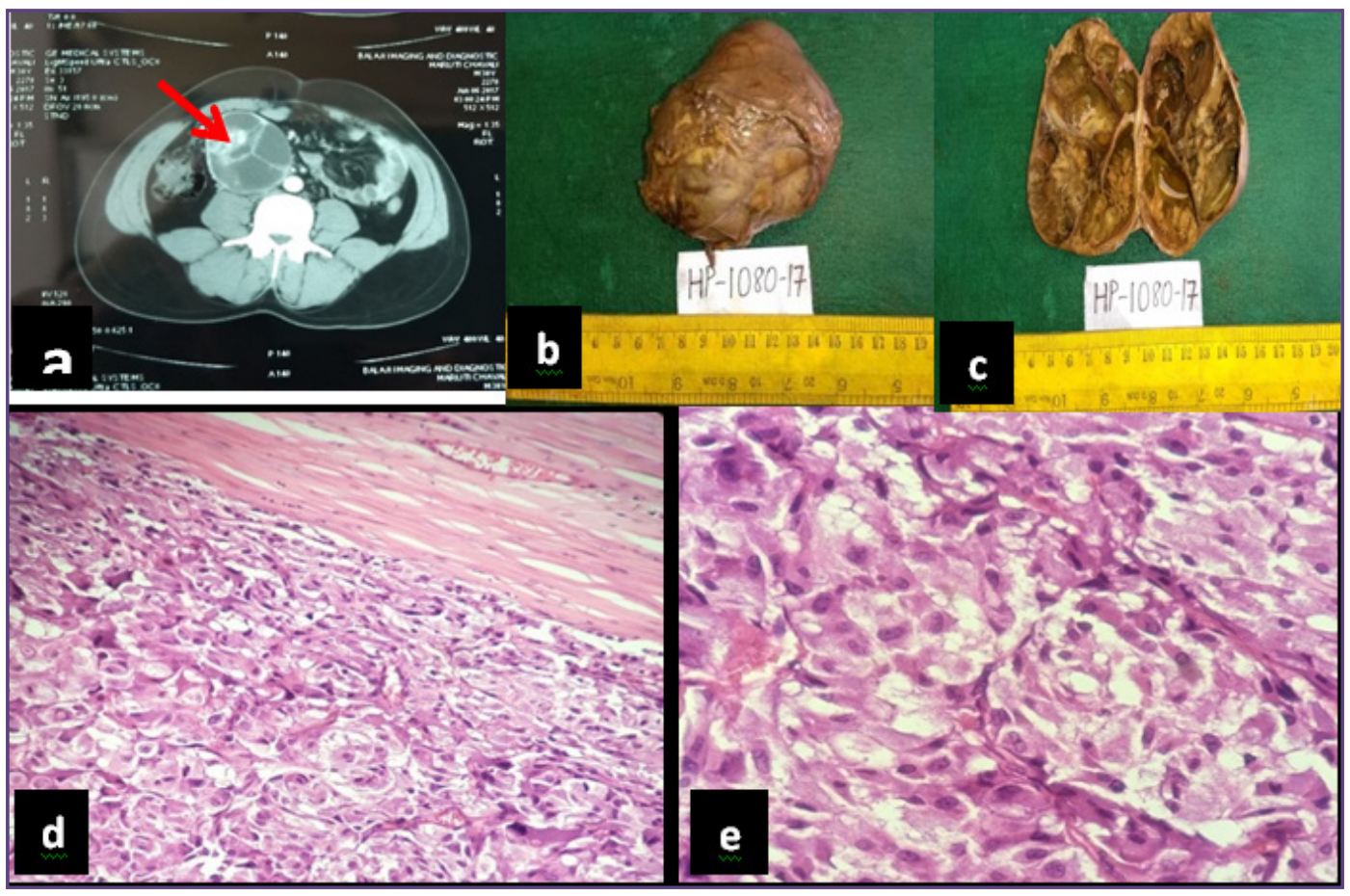

Fig. 1: CT scan(contrast enhanced) showing a)multiloculated cyst with partly solid areas . Gross photograph showing b) cystic mass with c) multiloculations and partly solid areas on cut section. Microphotograph showing d)tumor cells arranged in Zellballen pattern with e)abundant eosinophilic granular cytoplasm(H \& E,X400).

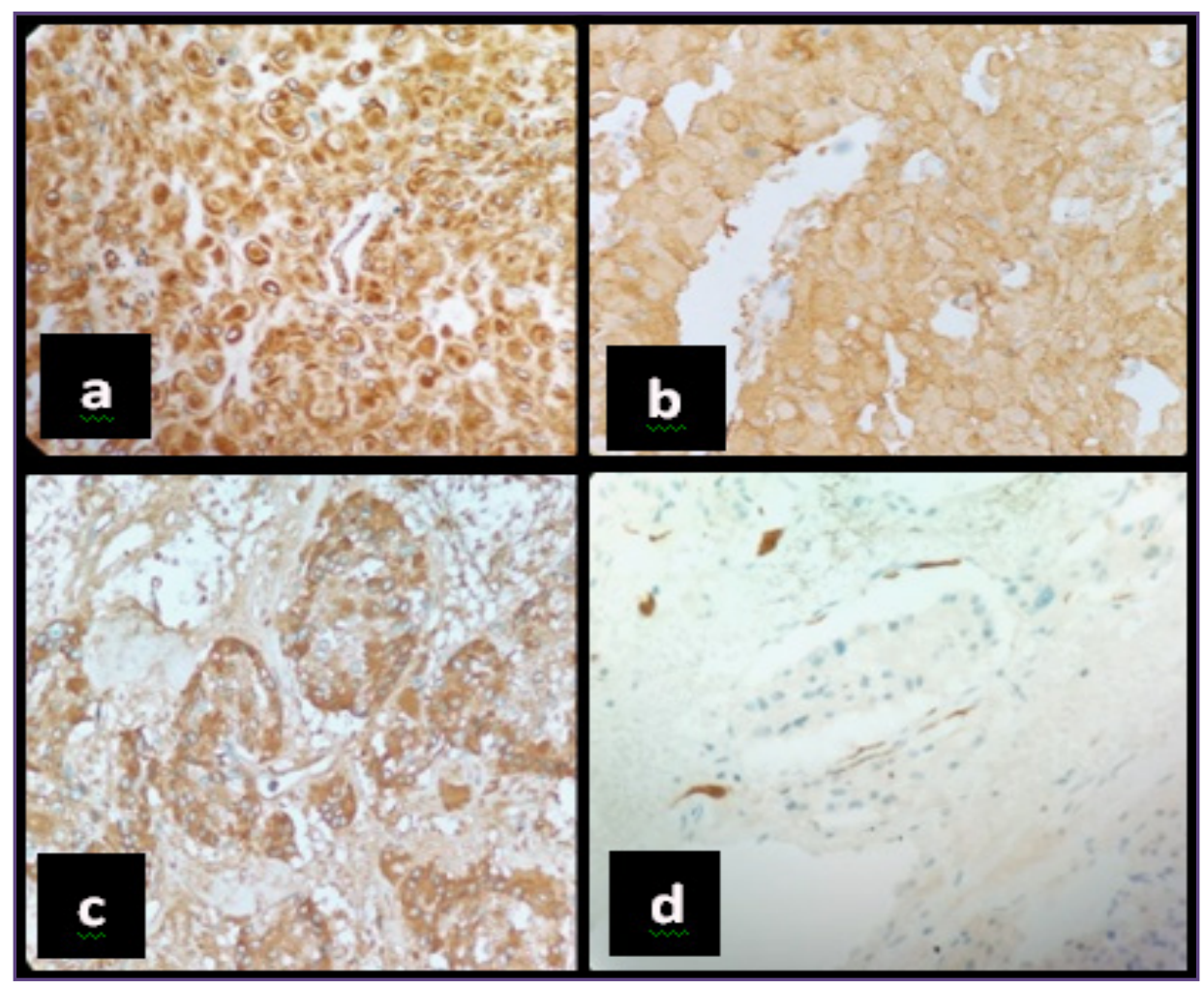

Fig. 2: Microphotograph showing tumor cells strongly positive for (a) vimentin, b)synaptophysin,(c)chromogranin with (d) focal S-100 positive sustentacular cells(IHC,X 400). 


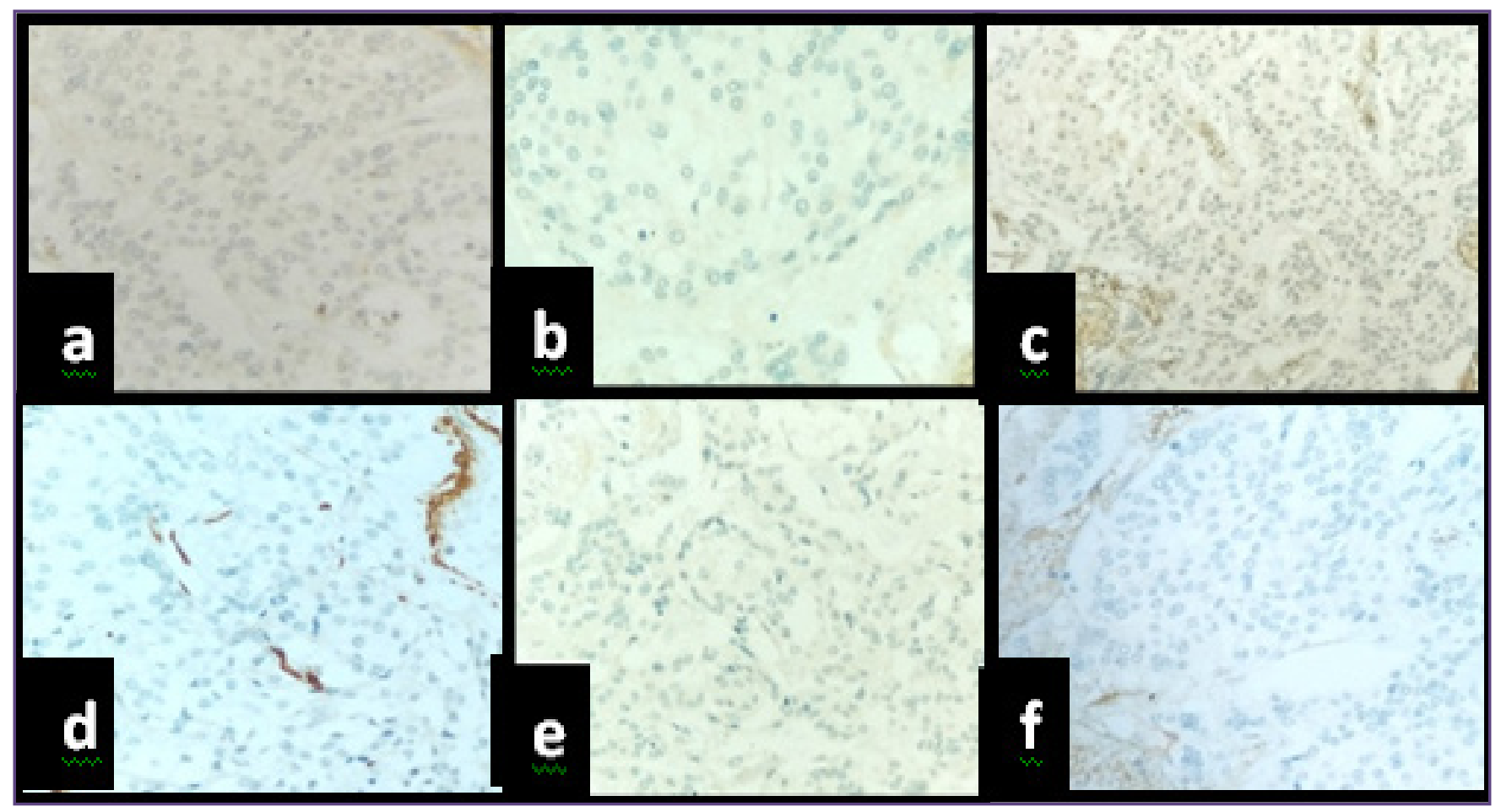

Fig. 3: Immunohistochemistry showing tumor cells negative for a)pancytokeratin,b)EMA,c)TFE3,d)MSA,e)PAX8, and f) CD10(IHC,X 400).

are considered to be hereditary. Furthermore, hereditary cases have also been associated with multiple endocrine neoplasia 2,Von Hippel-Lindau disease, Carney triad and neurofibromatosis type 1 and type 2; consequently,genetic testing should be considered for all patients diagnosed with paragangliomas. ${ }^{[3]}$ In our case though it was not associated with any syndromes genetic testing was not possible due to financial constraints.

The clinical manifestations of extradrenal paraganglioma are variable and nonspecific. Headache, palpitations and profuse sweating constitutes the classical triad of symptoms of paraganglioma ${ }^{[5]}$ At times, they are asymptomatic or present with abdominal pain and palpable mass .The radiological modalities and immunohistopathology helps in finally clinching the diagnosis and predicting its malignant potential. The following parameters may be taken into account for malignant potential and behaviour of paragangliomas: location, size, Pheochromocytoma of Adrenal Gland Scales Score(PASS) ,S-100 immunoreactivity and Ki-67 labelling index. ${ }^{[6]}$

In the present case, the tumor cells were positive for synaptophysin and chromogranin indicating that it was a neuroendocrine tumor. Pancytokeratin and EMA were negative, indicating nonepithelial tumor. TFE3, CD10,
PAX8 were negative which helped us to differentiate from renal cell carcinoma, pseudo papillary cyst of pancreas and carcinomas in general respectively.

The mainstay of treatment has been surgical removal. ${ }^{[2]}$ Chemotherapy has no defined role for the treatment of paraganglioma except in metastatic disease. Due to possibility of recurrence and metastasis especially after incomplete surgical excision, patients need periodic check and long term follow-up. ${ }^{[7]}$

Mesenteric paraganglioma, presenting as cystic mass in asymptomatic patient is a rare entity which can be confused with other abdominal cystic tumors. Careful assessment of histology, IHC evaluation and genetic testing are required for confirmation of diagnosis. Because of malignant or metastatic potential and high recurrence rate, pre and postoperative imaging study and lifelong follow-up is recommended. ${ }^{[8]}$

\section{Acknowledgements}

Nil

\section{Funding \\ Nil}

\section{Competing Interests}

Nil 


\section{Reference}

1. Kudoh A, Tokuhisa Y, Morita K, Hiraki S et al. Mesenteric Paraganglioma: Report of a Case. Surg Today. 2005; 35:594-97.

2. Granger J, Mahapatra R, Hamid B, Gillespie K et al. Incidental Mesenteric Paraganglioma: A Case Report and Literature review. Ann Coloproctol .2017;33(5):197-200.

3. Fujita T, Kamiya K, Takahashi Y, Migazaki S, Liho I, Kikuchi $\mathrm{H}$, et al. Mesenteric Paraganglioma: report of a case. World J gastrointestinal Surg. 2013;5:62-7.

4. Kok SYA, Leung CY, Chow KY. An unusual cause of back pain: A case of large nonfunctioning retroperitoneal paraganglioma presented as a large cystic lesion. A case report and review of literature. Journal of Surgical Case Reports. 2017;4:1-4.
5. Robbins, Cotran. Head and neck. In: Kumar, Abbas, Fausto, Aster. Pathologic basis of disease.8th edn. Haryana:Elsevier;2011.741-42.

6. Khandeparkar SGS, KulKarni MM,Gaopande V, Joshi A et al. An unusual case of paraganglioma of the broad ligament presenting as cystic mass. South Asian Journal of Cancer 2014;3:229-30.

7. Sreemani NK, Nandyala VNR, Srujana S, Quadri SSS, et al. Retroperitoneal composite pheochromocytoma/ paraganglioma complicating pregnancy a rare case report. Int Surg J 2015;2:85-7.

8. Beex L, Gennatas G, Goodman A, Razzouk BI, Romkes M. Retroperitoneal paraganglioma presenting as chest pain: a case report. Case Rep Oncol Med 2013;2013:329472.http:// dxdoi.org/10.1155/2013/329472.

*Corresponding author:

Dr. M. N. Jadhav, Associate Professor, Department of Pathology, Belagavi Institute of Medical Sciences, Belagavi-590001 INDIA

Email: shubhamj2003@yahoo.co.in

Date of Submission : 27/11/2019

Date of Acceptance : 13/04/2020

Financial or other Competing Interests: None.

Date of Publication : 27/06/2020 